\section{Comunicações persuasivas e doação regular de sangue: um estudo experimental}

\author{
Persuasive communications and regular blood \\ donation: an experimental study
}

\footnotetext{
${ }_{1}$ Universidade Federal da Paraíba, João Pessoa, Brasil.

Correspondência B. G. F. Cunha Universidade Federal da Paraíba.

Rua Geraldo Macário $S$. de Oliveira 244, João Pessoa, PB 58075-655, Brasil. balduino@cchla.ufpb.br
}

\begin{abstract}
This study aimed to: investigate yielding to the dependent variable "behavioral intent to become a regular blood donor", verify the impact of such communications on variance in the dependent variable, examine the single contribution of the external independent variable to the Rational Action Theory, and test the fit of the expanded Rational Choice Theory to the target behavior and sample. Only a post-test design and double-blinded procedure were used, randomly picking 405 university students for experimental groups 1 and 2, placebo control, and control only. The results showed: lack of yielding by the experimental groups; considerable percentage variance in the dependent variable explained by the independent variable in the experimental and placebo control groups; and satisfactory and significant correlations for variables in the expanded theory. Absence of yielding for the criterion variable was probably due to the time interval. The positive persuasive strategy accounted for the greatest variance in the dependent variable. Moral obligation showed the greatest impact on participants' intent to perform the behavior. The correlations corroborated the theoretical and methodological validity of the expanded theory.
\end{abstract}

Persuasive Communications; Blood Donors; Social Responsability; Behavior
Balduino Guedes Fernandes da Cunha 1

Mardonio Rique Dias 1

\section{Introdução}

No panorama mundial, as dificuldades para a obtenção regular e de boa qualidade de sangue têm preocupado peritos médicos, sanitários e sociais 1,2,3. Contribuem para esse quadro o fato de (1) as principais formas de doação de sangue às instituições hematológicas serem do tipo reposicional, seletiva ou autóloga - em detrimento àquela voluntária e regular - e (2) não participação significativa da população universitária enquanto contingente expressivo de potenciais doadores de sangue.

De forma similar, observa-se atualmente que a situação real da obtenção de sangue no sistema de saúde brasileiro (doação de reposição, sexo masculino, classe baixa, baixa escolaridade, doadores inaptos), em relação ao seu objetivo principal - obtenção regular e de boa qualidade de sangue - apresenta-se totalmente distante da situação ideal (doação espontânea e regular, ambos os sexos, todas as classes sociais, todos os níveis educacionais, doadores aptos), e explica em grande parte o déficit de oferta em prejuízo ao aumento da demanda nos hemocentros, bancos de sangue e hospitais ${ }^{3}$.

É consenso que a reversão do quadro de escassez estaria em promover a doação de sangue, de forma voluntária e sistemática. Na busca de explicações para os determinantes do comportamento de doar sangue estudos têm focalizado razões altruístas e humanitaristas 4,5,6,7, fatores 
relacionados às normas, pressões sociais e incentivos 8 , representatividade e custo do ato de doação para o doador ${ }^{9}$ e demais motivos múltiplos 10 .

Não obstante a compreensão das bases motivacionais, é importante considerar que a intenção de ajudar (e.g., doando sangue) nem sempre está correlacionada - direta, forte e significativamente - com o comportamento observável (e.g., de doar sangue), o que torna necessário identificar os fatores que influenciam ambos $11 . \mathrm{Na}$ busca desse escopo, a utilização de estratégias interventivas subsidiadas por modelos teóricos consistentes tem sido enfatizada por peritos sociais como uma forma promissora de compreender e modificar a conduta humana.

Nesta perspectiva, a Teoria da Ação Racional 12,13 é um dos modelos que tem sido utilizado para descrever, explicar, predizer e prescrever comportamentos volitivos de prevenção de doenças e de promoção da saúde 14,15,16,17,18,19,20, $21,22,23,24,25,26,27,28,29,30,31,32,33,34,35,36,37$. De acordo com o modelo, o comportamento da pessoa é determinado pela sua intenção comportamental de realizar este comportamento. Dois fatores principais determinam a intenção comportamental do indivíduo: um fator pessoal (sua atitude) e um fator social (sua norma subjetiva). Cada um desses fatores é também uma função de dois outros determinantes. A atitude da pessoa é uma função daquilo que ela crê que irá acontecer ao desempenhar o comportamento (suas crenças comportamentais) e das avaliações que ela faz a respeito das conseqüências da execução desse mesmo comportamento (suas avaliações das conseqüências). O segundo componente, norma subjetiva, é uma função daquilo que a pessoa acredita que os outros acham que ela deve fazer (suas crenças normativas) e da motivação para corresponder ao que os outros acham que ela deve fazer (suas motivações para concordar) 38,39,40,41,42,43.

A despeito de, na Teoria da Ação Racional 12,13 , somente dois fatores principais - a atitude e a norma subjetiva - permitirem a predição do comportamento por meio da intenção comportamental, a adição de um terceiro fator - representado pela obrigação moral - ao modelo causal básico é defendida como uma variável determinante das intenções, principalmente àquelas de natureza altruística ou pró-social 44.

A obrigação moral é entendida como um tipo de obrigação individualizada e internalizada que tem a função de fornecer ajuda aos outros em situações específicas, ativada em circunstâncias nas quais existe (1) ciência de que outras pessoas precisam de ajuda, (2) crença de que ações de ajuda existem, (3) senso de que se é capaz de desenvolver essas ações e (4) crença de que se é responsável pelo bem-estar daquelas pessoas que estão precisando de ajuda 3,45 . Ela tem influenciado comportamentos altruísticos do tipo cuidados com indivíduos deficientes ${ }^{45}$, cuidados com crianças abandonadas 46 e doação de órgãos 45,47. No caso da doação de sangue, estudos que analisaram a contribuição desta medida frente a demais variáveis, corroboraram-na como o principal determinante daquele comportamento 3,17,48,49,50. Nas investigações de Mello 3 e Cunha 17 é apresentada a Teoria da Ação Racional com a inclusão da variável externa obrigação moral (Teoria da Ação Racional ampliada).

No Brasil, em que pese a dificuldade de obtenção regular e voluntária de sangue, poucas pesquisas têm examinado o papel, em conjunto, da atitude, norma, obrigação moral e intenção como fatores determinantes deste comportamento 3,17 . Análises conjugadas dos processos empíricos relacionados aos contextos pessoais, sociais e cognitivos da doação de sangue também se mostram escassas 3,17,24,25,31.

Levando em conta tais considerações e utilizando a Teoria da Ação Racional com a adição da variável obrigação moral, Mello 3 levou a cabo uma investigação com 400 estudantes universitários com o objetivo de testar a aplicabilidade do modelo ampliado na explicação e predição da "intenção comportamental de tornar-se doador(a) regular de sangue". Em um primeiro momento, a autora realizou o levantamento das crenças sobre o comportamento de doar sangue e identificou aquelas crenças modais salientes, i.e, crenças eliciadas que corresponderam a uma freqüência maior ou igual a $75 \%$ do total de crenças emitidas pela amostra. Em um segundo momento, verificou que as variáveis preditoras obrigação moral, atitude, crença normativa geral e norma subjetiva explicaram, conjuntamente, $47,2 \%$ da variância da variável critério, intenção comportamental. Corroborando estudos anteriores, a variável externa obrigação moral, foi a que mais contribuiu na explicação da variância da variável efeito.

De posse dos resultados obtidos pela autora e (1) considerando as metas e os princípios expressos pela Política Nacional de Sangue, Componentes e Hemoderivados relativos ao incentivo à doação regular de sangue 51; (2) considerando, ainda, que no Brasil, a população universitária, por não representar um contingente expressivo de doadores, contribui para a situação de decréscimo da oferta de sangue em detrimento do aumento da sua procura; (3) considerando, também, que uma das formas mais eficientes de reverter esse quadro seriam campanhas de comunicação de massa (desenvolvidas, e.g., pelo Ministério da Saúde), incentivando a doação de 
sangue e; (4) considerando, por fim, que o modo de diligenciar tais mudanças comportamentais na população-alvo em prol da prática da doação de sangue seria elaborar e testar modelos de comunicações persuasivas alicerçadas nos fatores preditores da "intenção comportamental de tornar-se doador(a) regular de sangue" identificados por Mello 3, é que foi planejada e executada a pesquisa em questão, com os objetivos (e hipóteses empíricas associadas) elencados adiante.

\section{Objetivos e hipóteses}

Nesta seção e ulteriores foram observadas as normas sugeridas por Günther 52 e Ramos-Álvarez \& Catena 53 relativas, principalmente, à (1) adequabilidade e estrutura causal das hipóteses, (2) adequabilidade do desenho experimental, (3) operacionalização das variáveis dependente e independente, (4) validade e confiabilidade do instrumento de coleta de dados, (5) seleção probabilística da amostra e (6) condução ética da pesquisa.

Optou-se, também, pelo uso do termo yielding 12,13,17,18 no original, para caracterizar com precisão o efeito de rendição/capitulação dos participantes às mensagens persuasivas (variáveis independentes) sobre a adoção do comportamento investigado, i.é, a mudança da magnitude de suas intenções comportamentais (variável dependente) em direção à doação regular de sangue.

\section{Objetivo geral}

Verificar a presença (ou não) de yielding (capitulação) para os grupos experimentais devido à influência de comunicações persuasivas sobre a variável dependente "intenção comportamental de tornar-se doador(a) regular de sangue”.

\section{Hipótese geral}

O escore médio da variável dependente "intenção comportamental de tornar-se doador(a) regular de sangue" é significativamente maior para o grupo experimental 1, seguidos dos grupos experimental 2, controle-placebo e somente-controle.

\section{Objetivo específico 1}

Verificar o percentual de variância da variável conseqüente "intenção comportamental de tornar-se doador(a) regular de sangue" explicada conjuntamente pelas variáveis efeitos nas quatro condições de estudo.

\section{Hipótese específica 1}

A variância da variável conseqüente explicada conjuntamente pelas variáveis efeitos nos grupos experimentais é significativamente maior àquela explicada nos grupos controle-placebo e somente-controle.

\section{Objetivo específico 2}

Verificar o peso empírico da variável externa à Teoria da Ação Racional, obrigação moral, na predição da variável critério "intenção comportamental de tornar-se doador(a) regular de sangue”, nos grupos submetidos às mensagens persuasivas positiva e negativa.

\section{Hipótese específica 2}

A variável externa à Teoria da Ação Racional, obrigação moral, apresenta o maior peso empírico na predição da variável critério "intenção comportamental de tornar-se doador(a) regular de sangue", nos grupos submetidos às mensagens persuasivas positiva e negativa.

\section{Objetivo específico 3}

Testar o ajuste da Teoria da Ação Racional ampliada em descrever, explicar, predizer e prescrever o comportamento para a amostra investigada.

\section{Hipótese específica 3}

A Teoria da Ação Racional ampliada representa um modelo ajustado adequadamente na descrição, explicação, predição e prescrição do comportamento de tornar-se doador(a) regular de sangue para a amostra investigada.

\section{Método}

O método adotado seguiu as prescrições dos autores da Teoria da Ação Racional 12,13, bem como aquelas próprias para estudos de natureza experimental 53 .

\section{Etapa I: elaboração das comunicações persuasivas}

A construção das comunicações persuasivas seguiu as normas do modelo da Teoria da Ação Racional 12,13, sendo estas elaboradas nas versões positiva, negativa e irrelevante 17 . As duas primeiras comunicações tiveram por objetivo influenciar os componentes atitudinal, normativo e moral do comportamento tornar-se doador(a) 
regular de sangue, sendo as crenças selecionadas para a elaboração de ambas, aquelas crenças modais salientes que se mostraram mais discriminativas, no estudo de Mello ${ }^{3}$, entre os grupos de sujeitos que intencionavam e não intencionavam realizar o comportamento investigado. A comunicação positiva exortava as vantagens em se adotar o comportamento; a negativa, as desvantagens em não se adotar o comportamento.

As comunicações persuasivas irrelevantes foram elaboradas tendo por objetivo persuadir à adoção do uso racional da água. Para tanto, foram utilizadas informações sobre o (1) gerenciamento de recursos hídricos no Brasil e no mundo 54, (2) Projeto Brasil das Águas 55 e (3) ciclo hidrológico e gerenciamento integrado 56.

Etapa II: aplicação e estudo experimental das comunicações persuasivas

\section{- População e amostra}

Com base na população-alvo de 17.300 discentes universitários do campus I da Universidade Federal da Paraíba e com a adoção de um erro máximo e intervalo de confiança de 5\% e 95\%, respectivamente, foi calculada uma amostra probabilística, através do programa Stats versão 1.1 (Decision Analyst Inc., Arlington, Estados Unidos), por agrupamento sistemático composta por 405 participantes (média $=26,4$ anos e desvio-padrão- DP = 7,0; 43,2\% masculinos e 56,8\% femininos; $69,4 \%$ solteiros).

\section{- Desenho experimental}

Foi composto por um planejamento poligrupal (ampliação do delineamento no. 6 de Campbel \& Stanley 57), com distribuição equânime dos participantes $\left(\chi^{2}(3)=0,42\right.$; $\left.p>0,05\right)$ para as condições comunicação persuasiva positiva (grupo experimental 1 = 100 participantes), comunicação persuasiva negativa (grupo experimental $2=97$ participantes), comunicação persuasiva irrelevante (grupo controle-placebo = 102 participantes), sem comunicação persuasiva (grupo somente-controle $=106$ participantes), todas aleatoriamente constituídas e com medidas só pósteste. A escolha deste desenho atendeu a critérios de controle das principais fontes de invalidação metodológica interna e externa 57,58 .

\section{- Instrumento}

O instrumento utilizado para a mensuração dos efeitos das comunicações persuasivas sobre a variável critério foi aquele elaborado e validado por
Mello ${ }^{3}$ conforme prescrições da Teoria da Ação Racional - de formato estruturado e respostas a "lápis e papel" -, composto por 30 itens na forma de escalas bipolares - de diferencial semântico (bom-ruim, útil-inútil) e de probabilidade subjetiva (provável-improvável) - ancorados em um continuum de sete pontos para a medida de todas as variáveis do modelo, mais a variável externa obrigação moral. A apresentação e conclusão do instrumento seguiram as orientações de Günther 59 .

\section{- Procedimentos}

Duas auxiliares de pesquisa realizaram a identificação e a posterior listagem de todas as salas de aula (conglomerados). Com o auxílio do programa Stats versão 1.1, foram montados 16 agrupamentos compostos por cinco salas (aleatoriamente distribuídas) cada e, posteriormente, realizada a blocagem (também probabilisticamente) de quatro grupos de quatro agrupamentos para a composição de cada condição de estudo (com nova distribuição casual). Em momento ulterior, as auxiliares de pesquisa, estabeleceram o rapport ("clima" de confiança) com os participantes, explicaram-lhes os objetivos do estudo e solicitaram suas participações voluntárias.

Utilizou-se o procedimento duplo-cego para controlar vieses ou tendências das auxiliares de pesquisa e a desejabilidade social e característica da demanda por parte dos participantes. O grupo somente-controle foi o primeiro a ser contatado, vindo a seguir as condições controle-placebo, experimental 2 e experimental 1, nesta ordem, com o fito de se evitar a contaminação do efeito de conteúdo das comunicações persuasivas entre os participantes 57,58 .

Foram respeitadas as diretrizes e normas regulamentadoras de pesquisas envolvendo seres humanos, conforme rezam legislações específicas em vigor $60,61,62$.

\section{Resultados}

Nos tabelamentos e análises de resultados que se seguem utilizou-se o SPSS 11.0 (SPSS Inc., Chicago, Estados Unidos), através do qual foram eliminados aqueles participantes que apresentaram altos índices (cerca de 1/3) de missing values (valores omissos) para o total de itens do instrumento, bem como os que se apresentaram como outliers (respostas com valores extremos) 63,64 . Os resultados para o grupo experimental 1 (GE1 - comunicação persuasiva positiva), grupo experimental 2 (GE2 - comunicação persuasiva negativa), grupo controle-placebo (GCP - comu- 
nicação persuasiva irrelevante) e grupo somente-controle (GSC - sem comunicação persuasiva) são dispostos adiante.

\section{Análises das matrizes de correlações}

r de Pearson

Com a finalidade de verificar a força e o sentido das relações entre as variáveis do modelo da Teoria da Ação Racional ampliada 3,17, foram realizadas correlações simples produto-momento $r$ de Pearson, acompanhadas por seus respectivos níveis de significância (p) para as quatro condições de estudo (Tabela 1).

No GE1 foram encontradas correlações positivas variando de moderadas a relativamente fortes, todas significativas, entre a variável critério intenção comportamental e as variáveis independentes obrigação moral, crença normativa geral, atitude e crença comportamental geral. Aquela também se relacionou, de forma moderada e significativa com a norma subjetiva. É oportuno atentar para o fato da correlação observada entre a variável externa, obrigação moral, e a variável dependente, intenção comportamental, ter sido a maior em magnitude, seguida da crença normativa geral, da atitude e da crença comportamental geral, todas bastante significativas.

A matriz de correlações positivas, verificada no GE2, entre a intenção comportamental e as demais variáveis preditivas foram bastante significativas. Relações fortes foram encontradas para a obrigação moral, seguida da crença normativa geral e da crença comportamental geral. Correlações moderadas foram observadas para a norma subjetiva e para a atitude. Destaquese que a variável externa ao modelo original, obrigação moral, também apresentou correlação forte com a variável crença comportamental geral, seguida da variável critério intenção comportamental, da crença normativa geral e da atitude.

Tabela 1

Matriz de correlações $r$ de Pearson entre as variáveis da Teoria da Ação Racional ampliada.

\begin{tabular}{|c|c|c|c|c|c|c|}
\hline Grupos & Variáveis & AT & CCG & NS & CNG & OM \\
\hline Grupo experimental 1 & IC & 0,542 * & 0,538 * & 0,335 * & 0,639 * & 0,729 * \\
\hline \multirow[t]{4}{*}{ (comunicação persuasiva positiva; $n=99$ ) } & AT & - & 0,581 * & 0,105 n.s. & 0,408 * & $0,541 *$ \\
\hline & CCG & - & - & 0,154 n.s. & 0,508 * & $0,480 *$ \\
\hline & NS & - & - & - & 0,508 * & 0,297 ** \\
\hline & CNG & - & - & - & - & 0,556 * \\
\hline Grupo experimental 2 & IC & 0,467 * & 0,503 * & 0,474 * & 0,610 * & 0,649 * \\
\hline \multirow[t]{4}{*}{ (comunicação persuasiva negativa; $n=96$ ) } & AT & - & 0,769 * & 0,406 * & 0,432 * & 0,500 * \\
\hline & CCG & - & - & 0,429 * & 0,534 * & 0,659 * \\
\hline & NS & - & - & - & 0,624 * & 0,406 * \\
\hline & CNG & - & - & - & - & $0,602 *$ \\
\hline Grupo controle-placebo & IC & $0,246 * \star \star$ & $0,233 * \star \star$ & $0,278 * \star$ & $0,515 *$ & $0,687 *$ \\
\hline \multirow[t]{4}{*}{ (comunicação persuasiva irrelevante; $\mathrm{n}=101$ ) } & 1) $\mathrm{AT}$ & - & 0,491 * & $-0,170$ n.s. & 0,060 n.s. & 0,137 n.s. \\
\hline & CCG & - & - & $-0,104$ n.s. & $0,313 * \star \star$ & $0,397 *$ \\
\hline & NS & - & - & - & 0,492 * & 0,186 n.s. \\
\hline & CNG & - & - & - & - & 0,550 * \\
\hline Grupo somente-controle & IC & 0,374 * & $0,274 \star \star$ & $0,308 * \star$ & $0,543 *$ & $0,322 * \star$ \\
\hline \multirow[t]{4}{*}{ (sem comunicação persuasiva; $n=106$ ) } & AT & - & 0,443 * & 0,140 n.s. & $0,310 * \star$ & 0,365 * \\
\hline & CCG & - & - & 0,160 n.s. & 0,389 * & 0,506 * \\
\hline & NS & - & - & - & 0,652 * & $0,236 * * *$ \\
\hline & $\mathrm{CNG}$ & - & - & - & - & 0,426 * \\
\hline
\end{tabular}

n.s.: não significativa; IC: intenção comportamental (variável dependente); AT: atitude (variável independente); CCG: crença comportamental geral (variável independente); NS: norma subjetiva (variável independente); CNG: crença normativa geral (variável independente); OM: obrigação moral (variável independente externa à Teoria da Ação Racional).

* $\mathrm{p}<0,05$

** $\mathrm{p}<0,01$

$\star \star \star ~ p<0,001$. 
No GCP, correlações positivas, altas e significativas foram encontradas para a intenção comportamental com a crença normativa geral e com a obrigação moral. Note-se que variável externa, obrigação moral, foi aquela que se correlacionou mais fortemente com a intenção comportamental, seguida da crença normativa geral e da crença comportamental geral, todas também bastante significantes.

$\mathrm{Na}$ matriz de correlações positivas para o GSC, que não sofreu nenhum tratamento experimental, a variável dependente correlacionou-se significativamente com todas as variáveis antecedentes. Uma correlação forte foi encontrada com a crença normativa geral, seguida de correlações moderadas com a atitude, a obrigação moral e a norma subjetiva, finalizando com uma correlação fraca com a crença comportamental geral. Para a variável externa obrigação moral, houve uma correlação forte com a crença comportamental geral, moderada com a crença normativa geral, a atitude e a intenção comportamental e fraca com a norma subjetiva.

\section{Análises de regressões lineares múltiplas}

Com o objetivo de verificar a contribuição das variáveis independentes na explicação da variância da variável critério "intenção comportamental de tornar-se doador(a) regular de sangue”, foram realizadas análises de regressão linear múltipla, através do método stepwise (por etapas) com o cálculo dos pesos empíricos de cada variável independente na equação de regressão, representados pelos betas $(\beta)$ estandardizados (Tabela 2). Foram observados os principais pressupostos para a realização desse tipo de análise multivariada, a saber: razão entre números de sujeitos e o número de variáveis independentes, inspeção e detecção dos outliers, verificação da existência de multicolinearidade e presença de homocedasticidade 64 .

Os resultados apresentados pelo GE1 demonstraram que a variável externa, obrigação moral, foi a melhor preditora da "intenção comportamental de tornar-se doador(a) regular de sangue", explicando, sozinha, praticamente $60 \%$ $(\beta=0,544)$ da variância da variável critério. Com o incremento das variáveis crença normativa geral ( $\beta=0,223$ ) e crença comportamental geral ( $\beta=$ 0,186 ) responderam, em seu conjunto, por quase $67 \%$ da variância da intenção comportamental.

Os resultados do GE2 indicaram que a obrigação moral foi quem melhor explicou a variância da "intenção comportamental de tornar-se doador(a) regular de sangue", da ordem de $42 \%$ $(\beta=0,458)$, seguida da medida crença normativa geral, com aproximadamente $6 \%(\beta=0,322)$. Em seu conjunto, ambas ficaram responsáveis por 48\% da variância da variável critério.

Os resultados verificados no GCP demonstraram que a obrigação moral obteve o maior peso empírico $(\beta=0,573)$ na equação de regressão, ficando responsável por, aproximadamente, $53 \%$ da variância da intenção comportamental. Também compuseram a equação de regressão, a crença normativa geral $(\beta=0,232)$ e a atitude $(\beta=$ $0,152)$. In totum, as três variáveis responderam com 57\% da predição para a variável critério.

Os resultados apresentados pelo GSC demonstraram que somente a crença normativa geral $(\beta=0,445)$ e a atitude $(\beta=0,236)$ atuaram como preditoras da variável dependente. Com um incremento aproximado de $4 \%$ desta última, ambas explicaram em torno de $30 \%$ da variância. Nota-se que nesta condição, a obrigação moral não teve nenhum impacto enquanto variável preditora.

\section{Análise de variância unidirecional (one-way ANOVA)}

Com o objetivo de detectar ou não efeitos de capitulação (yielding) para os GE1 e GE2 quando comparados aos GCP e GSC, foi realizada uma one-way ANOVA. As médias dos escores da variável dependente para cada grupo de estudo estão sumarizadas na Tabela 3 .

De acordo com os resultados obtidos, embora o GE1 tenha apresentado um escore médio superior da intenção comportamental (média $=4,57$ ), este não foi estatisticamente diferente quando comparado aos demais grupos $\left(\mathrm{F}_{(3 ; 398)}=1,275\right.$; $\mathrm{p}>0,05)$, sendo desnecessário se realizar um post hoc. Detectou-se, entretanto, que a média da intenção comportamental do GCP foi a segunda maior em magnitude (média $=4,24$ ), seguida da média do GSC (média $=4,22$ ) e do escore médio do GE2 (média = 4,1).

\section{Análise dos testes qui-quadrado}

Com a finalidade de se comparar os percentuais de variância da variável efeito explicados pelas variáveis antecedentes, foram realizados testes qui-quadrado entre as quatro condições de estudo, conforme apresentado na Tabela 4.

Os resultados obtidos demonstraram que houve diferenças para as quatro condições de estudo, quando comparadas as variâncias explicadas da variável critério pelas variáveis efeitos $\left(\chi^{2}{ }_{(3)}=14,23 ; p<0,05\right)$. Contudo, essas diferenças significativas ficaram localizadas para o GSC com os GE1 $\left(\chi^{2}{ }_{(1)}=13,63 ; \mathrm{p}<0,001\right)$, GE2 $\left(\chi^{2}{ }_{(1)}=4,07\right.$; $\mathrm{p}<0,05)$ e GCP $\left(\chi^{2}(1)=8,3 ; \mathrm{p}<0,01\right)$, não sendo observadas diferenças significantes entre os GE1 
Regressão linear múltipla (método stepwise) para a variável dependente intenção comportamental de tornar-se doador(a) regular de sangue.

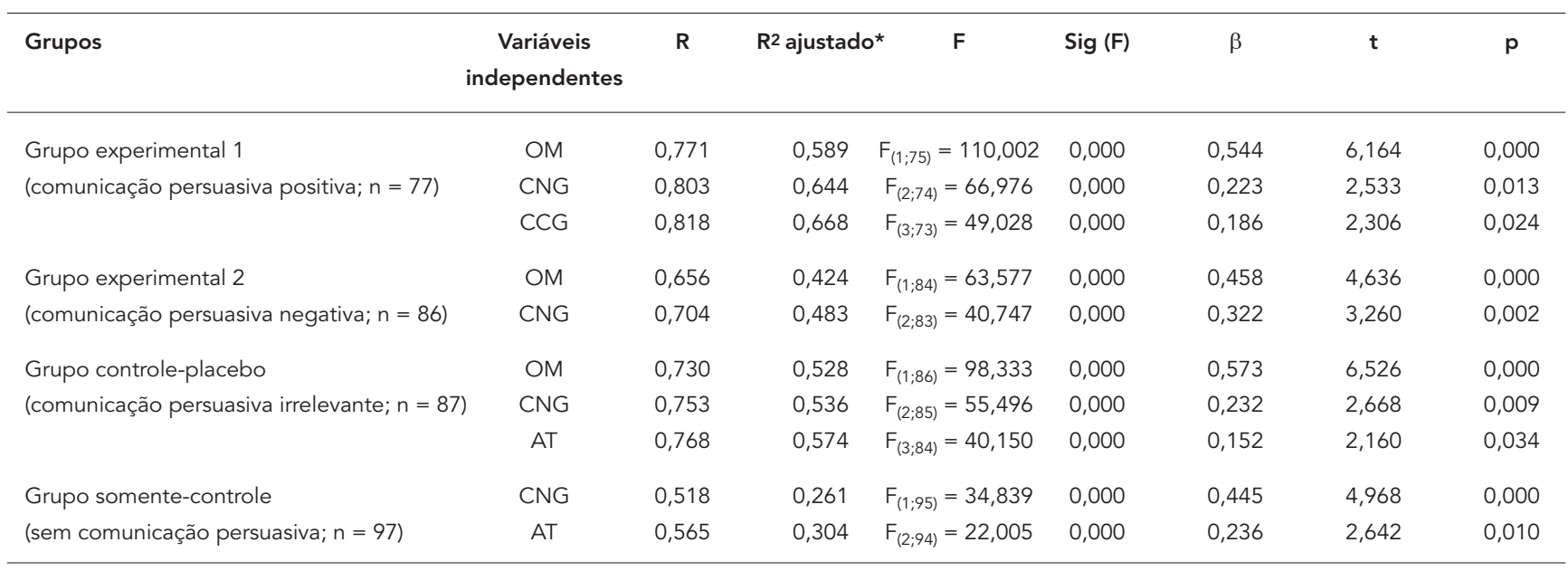

OM: obrigação moral; CNG: crença normativa geral; AT: atitude; CCG: crença comportamental geral.

* Valor da variância ajustado à predição na população.

e GE2 $\left(\chi^{2}{ }_{(1)}=2,97 ; \mathrm{p}>0,05\right)$ e entre o GCP com os GE1 $\left(\chi^{2}{ }_{(1)}=0,71 ; p>0,05\right)$ e GE2 $\left(\chi^{2}{ }_{(1)}=0,78\right.$; $\mathrm{p}>0,05)$.

\section{Discussão e conclusão}

Com relação ao objetivo geral deste trabalho e a hipótese probanda geral associada, os resultados observados (conforme Tabela 3) não evidenciaram a presença de yielding (capitulação) para os GE1 e GE2, corroborando a hipótese de nulidade.

Duas explicações podem ser aventadas nesse caso: (1) de acordo com o modelo da Teoria da Ação Racional 12,13 original, dentre os quatro níveis de especificidade a serem levados em conta na mensuração da variável conseqüente, enquanto a ação, o alvo e o contexto foram os mesmos neste estudo, o mesmo não aconteceu com relação ao tempo. É sensato admitir que no hiato temporal - aproximadamente quatro anos - verificado entre o estudo de Mello ${ }^{3}$ (no qual foram levantadas e identificadas as crenças modais salientes originais) e o presente, novas crenças modais salientes tenham sido formuladas pelos participantes. Desta forma, foi minimizada a correspondência esperada entre aquelas crenças originais (que subsidiaram o texto das comunicações persuasivas positiva e negativa) e o comportamento aqui investigado, reduzindo a influência das manipulações das variáveis antecedentes sobre a variável critério e, conseqüentemente, favorecendo a ausência de efeitos distintos significativos entre os quatro grupos de estudo; (2) ainda que as diferenças entre os grupos, para a variável dependente, não tenham apresentado magnitudes significantes, detectouse que o seu escore médio na condição de estudo comunicação persuasiva negativa (GE2) foi o menor em magnitude, dentre as quatro médias observadas. Este resultado pareceu sugerir que as admoestações no sentido das desvantagens em não adotar o comportamento de tornar-se doador(a) regular de sangue provocaram, entre os participantes deste grupo, um impacto para não agir favoravelmente ao comunicado persuasivo. Caberia inquirir: estaria presente nesse caso um provável efeito de reatância psicológica, da forma como Varela 65 enuncia? Uma replicação do estudo experimental aqui realizado, com o controle mais rigoroso das variáveis exógenas (notadamente àquelas relacionadas à validade metodológica interna e externa) e com a adição de itens aos instrumentos originais para identificar e mensurar o fenômeno da reatância psicológica para os grupos submetidos aos comunicados persuasivos, seria o procedimento mais adequado em investigações ulteriores como forma de responder satisfatoriamente esta questão.

Com alusão ao teste experimental das comunicações persuasivas (primeiro objetivo específico) e a hipótese experimental específica associada, os resultados apresentados na Tabela 4 corroboraram-na parcialmente e permitiram concluir que: (1), o grupo submetido à comunicação persuasiva positiva explicou maior variância da intenção comportamental que os grupos sub- 
Análise de variância unidirecional entre as médias da variável critério intenção comportamental de tornar-se doador(a) regular de sangue dos quatro grupos $(n=402)$.

\begin{tabular}{cccc}
\hline & Condição de estudo & \\
\hline $\begin{array}{c}\text { Grupo experimental 1 } \\
\text { (comunicação }\end{array}$ & $\begin{array}{c}\text { Grupo experimental } 2 \\
\text { persuasiva positiva) }\end{array}$ & Grupo controle-placebo & Grupo somente-controle \\
4,57 & persuasiva negativa) & (comunicação & (sem comunicação \\
persuasiva irrelevante) & 4,24 & 4,22 \\
$F_{(3 ; 398)}=1,275 *$
\end{tabular}

${ }^{*} p>0,05$

Teste qui-quadrado para percentuais de variância da variável dependente intenção comportamental de tornar-se doador(a) regular de sangue explicados pelas variáveis independentes dos quatro grupos ( $n=347)$.

\begin{tabular}{|c|c|c|c|c|}
\hline \multicolumn{4}{|c|}{ Condição de estudo } & \multirow[t]{2}{*}{$\chi^{2}$} \\
\hline Grupo experimental 1 & Grupo experimental 2 & Grupo controle-placebo & Grupo somente-controle & \\
\hline (comunicação & (comunicação & (comunicação & (sem comunicação & \\
\hline persuasiva positiva) & persuasiva negativa) & persuasiva irrelevante) & persuasiva) & \\
\hline $66,8 a$ & $48,3 a$ & $57,4 a$ & $30,4 b$ & $\chi^{2}(3)=14,23$ * \\
\hline
\end{tabular}

* $\mathrm{p}<0,005$.

Nota: os valores apresentando uma letra em comum não são significativamente diferentes a um $p<0,05$.

metidos às comunicações persuasivas negativa $\mathrm{e}$ irrelevante, contudo, de forma não significativa; (2) o grupo sob influência da mensagem persuasiva irrelevante explicou maior variabilidade da variável critério por parte das variáveis antecedentes, que o grupo sob influência do comunicado persuasivo negativo, também, de forma não significante; (3), comparadas com o grupo somente-controle (condição sem comunicação persuasiva), as variáveis independentes dos GE1 e GE2 e também do grupo controle-placebo, explicaram maior variância da intenção comportamental, nesse caso, de forma significativa.

Diante do que foi exposto, o conteúdo da mensagem persuasiva positiva aventa ser mais influente na variância da intenção comportamental, uma vez que as admoestações são no sentido de denotar as vantagens em tornar-se doador(a) regular de sangue. Entretanto, o conteúdo da mensagem persuasiva irrelevante, versando sobre um tema alheio à doação de sangue - utilização racional da água - mostrou-se, de um ponto de vista qualificativo, ser o segundo em poder de influência da variância da intenção comportamental. Uma explicação plausível para esse resultado estaria no fato de admoestações no sentido de alertar sobre as desvantagens em não adotar o comportamento de doação de sangue (como foi o caso da mensagem persuasiva negativa, a terceira em variabilidade de explicação) não teriam a mesma força que mensagens enfatizando as vantagens ou mesmo naquelas com um conteúdo de cunho ecológico (como foi o caso da mensagem persuasiva irrelevante), no qual se ressaltaram noções de dever e obrigação do uso racional da água que, de certa forma, também influenciaram um senso de dever e obrigação gerais em tornar-se doador(a) regular de sangue.

Atente-se para o fato de que o emprego da comunicação persuasiva irrelevante era evitar qualquer correspondência significante entre o seu conteúdo e o comportamento-alvo - i.é, ser de fato, irrelevante - atuando como um controle para o efeito placebo. Contudo, a evidência aqui observada, de que este comunicado influenciou de forma considerável a variabilidade da variável dependente, parece sugerir que mensagens específicas exortando à racionalização do uso de alguns recursos naturais têm um efeito indireto sobre alguns comportamentos altruísticos. Devido às implicações desta evidência, sua corro- 
boração por estudos independentes se revelaria pertinente para trabalhos ulteriores que versem sobre a adoção de comportamentos pró-sociais/ altruísticos e ecológicos/ambientais, detalhando-se a contribuição do fator moral enquanto seus determinantes.

Referente ao segundo objetivo específico e a hipótese experimental específica associada, esta foi corroborada, como demonstraram os resultados da Tabela 2, na qual podem ser verificados que os pesos empíricos - representados pelos betas estandardizados $(\beta)$ - da obrigação moral foram os maiores em magnitude nas equações de regressão das duas condições experimentais de estudo: 0,544 (para GE1) e 0,458 (para GE2).

Estes achados corroboraram estudos anteriores que têm demonstrado a importância da obrigação moral enquanto melhor preditora do comportamento de tornar-se doador(a) de sangue $3,48,49,50$. Ao que aquelas (e esta pesquisa) indicam, devido a sua natureza altruística e humanitária, o comportamento de doar sangue responde com mais força a argumentos nos quais a persuadibilidade se concentre em um senso de dever moral. Nesse ínterim, os resultados aqui obtidos evidenciaram que a obrigação moral teve maior impacto na decisão dos participantes em desempenhar o comportamento em questão, sugerindo sua incorporação ao conteúdo de campanhas sistemáticas que visem incentivar a doação periódica de sangue.

Atendendo ao terceiro e último objetivo específico deste trabalho e à hipótese alternativa específica associada, rejeitou-se a hipótese de nulidade dado que as correlações (simples e múltiplas) observadas para as quatro condições estudadas (conforme Tabelas 1 e 2) permitiram corroborar empiricamente a validade teórica e metodológica da Teoria da Ação Racional ampliada 3,17, ao demonstrar sua consistência interna e externa, bem como sua operacionalidade e parcimônia em descrever, explicar, predizer e prescrever o comportamento de doar sangue regularmente entre discentes universitários.

Contudo, faz-se oportuno aqui lembrar que a corroboração do modelo ampliado da Teoria da Ação Racional 3,17 confere-lhe somente o veredicto provisório de ajustamento em analisar o comportamento e amostra aqui investigados. Dessa forma, procurou-se cumprir neste último objetivo, uma das etapas fundamentais à lógica da investigação científica, qual seja, a de buscar a refutabilidade do corpus teórico trabalhado, com vistas a separar-lhe do rol de teorias especulativas e fantasiosas e demonstrar seus limites empíricos 66,67.

\section{Considerações finais}

De posse dos resultados, não foi possível determinar-para além de uma dúvida razoável-qual, dentre as estratégias persuasivas testadas, seria aquela mais eficiente. No entanto, há evidências razoavelmente fortes de que a abordagem calcada em argumentos positivos, vinculada a um senso de dever moral, mostre-se como a mais promissora em estratégias de comunicação em massa para públicos-meta específicos (como vem a ser o de discentes universitários, alvos desta investigação), com o objetivo de maximizar o número de doadores(as) regulares de sangue.

Importante considerar que a avaliação de tais estratégias precisa ser realizada, sistematicamente, através de um planejamento rigoroso, preferencialmente do tipo antes-e-depois. Não obstante isso, a necessidade do suporte de um referencial teórico refutável (comumente negligenciado nas comunicações massivas) é condição fundamental para se proceder de forma parcimoniosa e inteligível as devidas explicações pelo fracasso (ou mesmo, aparente sucesso) das campanhas institucionais de incentivo à doação de sangue. 


\section{Resumo}

Esta investigação teve por objetivos: verificar capitulação para a variável dependente "intenção comportamental de tornar-se doador (a) regular de sangue", averiguar influência destas comunicações na variância da variável dependente, examinar a contribuição isolada da variável independente externa, à Teoria da Ação Racional e testar o ajuste da Teoria da Ação Racional ampliada para o comportamento e amostra estudados. Utilizou-se delineamento só pós-teste e procedimento duplo-cego, designando-se aleatoriamente 405 estudantes universitários, para os grupos experimental 1, experimental 2, controle-placebo e somente-controle. Resultados demonstraram: inexistir capitulação para os grupos experimentais; consideráveis percentuais de variância da variável dependente explicados pelas independentes dos grupos experimentais e controleplacebo; correlações satisfatórias e significativas para variáveis da teoria ampliada. A ausência de capitulação para a variável critério deveu-se, provavelmente, ao intervalo do tempo. Coube à estratégia persuasiva positiva maior variância da variável dependente. A obrigação moral demonstrou maior influência na intenção dos participantes em desempenhar o comportamento. Correlações corroboraram validades teórica e metodológica da teoria ampliada.

Comunicações Persuasivas; Doadores de Sangue; Responsabilidade Social; Comportamento

\section{Colaboradores}

Ambos os autores participaram da concepção do projeto e elaboração do método (amostragem, desenho, instrumentos, procedimentos) da investigação, bem como das análises estatísticas pertinentes aos objetivos e hipóteses associadas. Coube a B. G. F. Cunha a redação final do artigo, após revisão de M. R. Dias.

\section{Agradecimentos}

Os autores agradecem às auxiliares de pesquisa Luciana da Silva Santos e Izayana Pereira Feitosa pelos criteriosos trabalhos de identificação/listagem dos conglomerados e de levantamento de dados da investigação; à Srta. Renata Lima de Madruga e ao Sr. Rogério Magalhães Guedes pelos competentes apoios logísticos; aos(às) pareceristas dos Cadernos de Saúde Pública pelas sugestões de aprimoramento do manuscrito; por fim, à Coordenação de Aperfeiçoamento de Pessoal de Nível Superior (CAPES) e ao Conselho Nacional de Desenvolvimento Científico e Tecnológico (CNPq) pelos apoios financeiros, respectivamente, na forma de bolsa de estudo do Programa de Demanda Social, ao primeiro autor e de bolsa de produtividade em pesquisa, ao segundo.

\section{Referências}

1. Chliaoutakis J, Trakas D, Socrataki F. Blood donor behaviour in Greece: implications for health policy. Soc Sci Med 1994; 38:1461-7.

2. Sarason IG, Sarason BR, Pierce GR, Shearin EN. A social learning approach to increasing blood donations. J Appl Soc Psychol 1991; 21:896-918.

3. Mello CC. Tornar-se doador(a) regular de sangue: predizendo o comportamento através da intenção comportamental [Dissertação de Mestrado]. João Pessoa: Curso de Pós-Graduação em Psicologia, Universidade Federal da Paraíba; 2000.

4. London P, Hemphill B. The motivations of blood donors. Transfusion 1965; 5:559-68.

5. Osborne D, Bradley S. Blood donor and nondonor motivation: a transnational replication. J Appl Psychol 1975; 60:409-10.
6. Oswalt R. A review of blood donor motivation and recruitment. Transfusion 1977; 17:123-35.

7. Oswalt R, Napolielo M. Motivations of blood donors and nondonors. J Appl Psychol 1974; 59:122-4.

8. Condie S, Warner W, Gillman D. Getting blood from collective turnips: volunteer donation in mass blood drives. J Appl Psychol 1976; 61:290-4.

9. Batson C, Ducan B, Ackerman P, Burckley T, Birch $\mathrm{K}$. Is empathic emotion a source of altruistic motivation? J Pers Soc Psychol 1981; 40:290-302.

10. Piliavin JA, Callero PL, Evans DE. Addiction to altruism? Opponent-process theory and habitual blood donation. J Pers Soc Psychol 1982; 43: 1200-13. 
11. Fishbein M, Jaccard J. Theorical and methodological issues in the predictin of family planning intentions and behaviors. Represent Res Soc Psychol 1973; 4:37-52.

12. Ajzen I, Fishbein M. Understanding attitudes and predicting social behavior. Upper Saddle River: Prentice Hall; 1980.

13. Fishbein M, Ajzen I. Belief, attitudes, intention and behavior: an introduction to theory and research. Reading: Adison-Wesley Publishing Company; 1975.

14. Bastos I. Intenção do tabagismo pós-conhecimento do enfisema pulmonar [Dissertação de Mestrado]. Rio de Janeiro: Curso de Pós-Graduação em Psicologia, Universidade Gama Filho; 2000.

15. Bezerra AE. Bebidas alcoólicas no contexto universitário: investigação fundamentada na Teoria da Ação Racional [Dissertação de Mestrado]. João Pessoa: Curso de Pós-Graduação de Enfermagem em Saúde Pública, Universidade Federal da Paraíba; 1997.

16. Brubaker RG, Wickersham D. Encouraging the practice of testicular self-examination: a field application of the Theory of Reasoned Action. Health Psychol 1990; 9:154-63.

17. Cunha BGF. Efeitos de comunicações persuasivas sobre a intenção comportamental de tornar-se doador(a) regular de sangue: um estudo experimental [Dissertação de Mestrado]. João Pessoa: Programa de Pós-Graduação em Psicologia Social, Universidade Federal da Paraíba; 2004

18. Dias MR. AIDS, comunicação persuasiva e prevenção: uma aplicação da Teoria da Ação Racional [Tese de Doutorado]. Brasília: Programa de PósGraduação em Psicologia Social e do Trabalho, Universidade de Brasília; 1995.

19. Dias MR, Lucena AAS. SIDA: exclusión social y desastre. Desastres y Sociedad 1997; 8:130-43.

20. Dias MR, Cavalcanti APR. Psicologia e nutrição: predizendo a intenção comportamental de aderir a dietas de redução de peso entre obesos de baixa renda. Estud Psicol (Natal) 2005; 10:121-9.

21. Formiga AC. A prática da caminhada na prevenção do infarto: uma investigação baseada na Teoria da Ação Racional [Dissertação de Mestrado]. João Pessoa: Curso de Pós-Graduação em Psicologia Social, Universidade Federal da Paraíba; 2002.

22. Gonçalves SMCM, Dias MR. A prática do autoexame da mama em mulheres de baixa renda: um estudo de crenças. Estud Psicol (Natal) 1999; 4:141-59.

23. Gonçalves SMCM. Fatores preditores da intenção de praticar o auto-exame da mama em mulheres de baixa renda [Dissertação de Mestrado]. João Pessoa: Curso de Pós-Graduação em Psicologia Social, Universidade Federal da Paraíba; 1998.

24. Lima VM. Efeitos de uma comunicação persuasiva sobre as crenças, atitudes e intenções de estudantes universitários acerca de se cadastrar como doador voluntário e periódico de sangue: uma aplicação da teoria de Fishbein e Ajzen [Dissertação de Mestrado]. Brasília: Curso de Pós-Graduação em Psicologia Social e do Trabalho, Universidade de Brasília; 1982.
25. Lima VM, D'Amorim MA. Aplicação da teoria da persuasão de Fishbein e Ajzen no recrutamento de doadores voluntários e periódicos de sangue. Arq Bras Psicol 1985; 37:110-24.

26. Marchtein R. A influência de uma mensagem persuasiva baseada no modelo da Teoria da Ação Racional no comportamento alimentar saudável [Dissertação de Mestrado]. Rio de Janeiro: Curso de Pós-Graduação em Psicologia, Universidade Gama Filho; 1999.

27. Norman NM, Tedeschi JT. Self-presentation, reasoned action, and adolescent's decisions to smoke cigarettes. J Appl Soc Psychol 1989; 19:543-58.

28. Oliveira SHS, Dias MR, Silva MIT. Adolescentes e AIDS: fatores que influenciam a intenção de uso do preservativo. DST J Bras Doenças Sex Transm 2005; 17:32-8.

29. Rise J. Predicting the intention to eat healthier food among young adult. Health Educ Res 1996; 10:453-61.

30. Saldanha AAW. Aspectos psicossociais de prevenção da AIDS em mulheres de baixa renda: entre o querer e o poder [Dissertação de Mestrado]. João Pessoa: Curso de Pós-Graduação em Psicologia Social, Universidade Federal da Paraíba; 1998.

31. Santanna M. Doação de sangue voluntária e periódica: uma aplicação da Teoria da Ação Racional [Dissertação de Mestrado]. Rio de Janeiro: Curso de Pós-Graduação em Psicologia, Universidade Gama Filho; 2005.

32. Schlegel RP, D'Avernas JJ, Zanna MD, DeCourville NH, Manske SR. Problem drinking: a problem for the Theory of Reasoned Action? J Appl Soc Psychol 1992; 22:358-85.

33. Soares JG. Fatores preditores da intenção de amamentar seus filhos em gestantes de baixa renda [Dissertação de Mestrado]. João Pessoa: Curso de Pós-Graduação em Psicologia Social, Universidade Federal da Paraíba; 1999.

34. Sousa FC. Fatores determinantes da intenção comportamental de pedir para o parceiro usar a camisinha: um estudo de persuasão com mulheres de baixa renda [Dissertação de Mestrado]. João Pessoa: Curso de Pós-Graduação em Psicologia Social, Universidade Federal da Paraíba; 2000.

35. Sutherland HJ, Cunha R, Lookwood G, Till JE. What attitudes and beliefes underlie patient's decisions about participating in chemotherapy trials? Med Decis Making 1998; 18:61-9.

36. Valois P, Desharnais R, Godin G. A comparison of the Fishbein and Ajzen and Triandis attitudinal models for prediction of exercise intention and behavior. J Behav Med 1988; 11:459-72.

37. Wallace KG, Koeppel K, Senko A, Stawiaz K, Thomas C, Kosar K. Effect of attitudes and subjective norms on intention to provide oral care to patients receiving antineoplastic chemotherapy. Cancer Nurs 1997; 20:34-41.

38. D’Amorim MA. A Teoria da Ação Racional e sua aplicação a comportamentos de saúde no Brasil. Mente Social 1999; 5:37-53.

39. D’Amorim MA. A Teoria da Ação Racional. Revista Ciências Humanas 1995; 30:92-106. 
40. D’Amorim MA. A medida da Teoria da Ação Racional. In: Pasquali L, organizador. Teoria e métodos de medida em ciências do comportamento. Brasília: Instituto Nacional de Estudos e Pesquisas Educacionais; 1996. p. 367-86.

41. D'Amorim MA, Vera VMLM. A relação atitude/ comportamento à luz da Teoria da Ação Racional. Arq Bras Psicol 1996; 35:133-42.

42. Dela Coleta MF, organizadora. Pesquisa e modificação de comportamento de saúde. Taubaté: Cabral Editora e Livraria Universitária; 2004.

43. Tuck M. Como escolhemos? - Psicologia do consumidor. Rio de Janeiro: Jorge Zahar Editor; 1978.

44. Schwartz S, Tessler R. A test of model for reducing measured attitude-behavior discrepancies. J Pers Soc Psychol 1972; 24:225-36.

45. Ruston JP, Sorretino RM. Altruism and helping behavior: social, personality, and developmental perspectives. Hillsdale: Erlbaum Associates; 1981.

46. Berkowitz L. Advances in experimental social psychology. New York: Academic Press; 1977.

47. Fellner MD, Schwarts SH. Altruism in disrepute: medical versus public attitudes toward the living organ donor. N Engl J Med 1971; 284:582-5.

48. Kaloupec DG, White H, Wong M. Multiple assessments of coping strategies used by volunteer blood donation: implications for preparatory training. J Behav Med 1984; 7:35-60.

49. Pomazal RJ, Jaccard JJ. An informational approach to altruistic behavior. J Pers Soc Psychol 1976; 33:317-26.

50. Zuckerman M, Reis HT. Comparison of three models for predicting altruistic behavior. J Pers Soc Psychol 1978; 36:498-510.

51. Brasil. Lei no. 10.205. Regulamenta o $\S 4^{\circ}$ do art. 199 da Constituição Federal, relativo à coleta, processamento, estocagem, distribuição e aplicação do sangue, seus componentes e derivados, estabelece o ordenamento institucional indispensável à execução adequada dessas atividades, e dá outras providências. Diário Oficial da União 2001; 21 mar.

52. Günther H. [Auto]-avaliação de um relato ou projeto de pesquisa. Brasília: Laboratório de Psicologia Ambiental, Universidade de Brasília; 2003. (Série Planejamento de Pesquisa nas Ciências Sociais, 6).

53. Ramos-Álvarez MM, Catena A. Normas para elaboración y revisión de artículos originales experimentales en ciencias del comportamiento. Rev Int Psicol Clín Salud 2003; 4:173-89.
54. Setti AA, Lima JEFW, Chaves AGM, Pereira IC. Introdução ao gerenciamento de recursos hídricos. Brasília: Agência Nacional de Energia Elétrica; 2000

55. Agência Nacional de Águas. Projeto Brasil das Águas. http://www.brasildasaguas.com.br (acessado em 18/Fev/2003).

56. Tundisi JG. Ciclo hidrológico e gerenciamento integrado. Ciênc Cult (São Paulo) 2003; 4:31-3.

57. Campbel DT, Stanley JC. Delineamentos experimentais e quase-experimentais de pesquisa. São Paulo: Editora da Universidade de São Paulo; 1979.

58. Escotet MA. Diseño multivariado en psicología y educación. Barcelona: Ediciones Ceac; 1980.

59. Günther H. Como elaborar um questionário. In Pasquali L, organizador. Instrumentos psicológicos: manual prático de elaboração. Brasília: Laboratório de Pesquisa em Avaliação e Medida, Instituto Brasileiro de Avaliação e Pesquisa em Psicologia; 1999. p. 231-58.

60. World Medical Association. Declaration of Helsinki. http://www.wma.net/e/policy/b3.htm (acessado em 02/Jul/2003).

61. Conselho Nacional de Saúde. Resolução CNS no. 196/96. Aprova as diretrizes e normas regulamentadoras de pesquisas envolvendo seres humanos. http://conselho.saude.gov.br (acessado em 02/ Jul/2003).

62. Conselho Federal de Psicologia. Resolução CFP no. 016/2000. Dispõe sobre a realização de pesquisa em psicologia com seres humanos. http://www. pol.org.br (acessado em 02/Jul/2003).

63. Bryman A, Cramer D. Análise de dados em ciências sociais: introdução às técnicas utilizando o SPSS. Oeiras: Celta Editora; 1992.

64. Tabachnick B, Fidell LS. Using multivariate statistic. New York: Harper Collins Publishers; 1996.

65. Varela JA. Soluções psicológicas para problemas sociais: uma introdução à tecnologia social. São Paulo: Editora Cultrix; 1974.

66. Popper KR. Conjecturas e refutações. Brasília: Editora da Universidade de Brasília; 1982.

67. Popper KR. A lógica da pesquisa científica. São Paulo: Editora Cultrix; 1972.

Recebido em 27/Fev/2007

Versão final reapresentada em 03/Set/2007

Aprovado em 08/Nov/2007 